\title{
Les facteurs influençant la participation des femmes rurales aux activités agricoles
}

\author{
Benali Amina, \\ Faculté des sciences économiques et sciences de gestion Université Tahari Mohamed, Béchar. Algérie.
}

Received October 10, 2016; Decembre 24, 2016; Published Online 2016/12/31

Copyright (c) 2016, African Journal of Applied Statistics (AJAS) and Probability African Society (SPAS). All rights reserved

\begin{abstract}
The aim of this study is to explore the determiners of the participation of the women in the agricultural activities. The data of the survey (investigation) collected with 678 rural women living on the West Algerian. A probit model was used to estimate the contribution of every determiner on the participation of the rural women to the agricultural activities. The results indicate that the age of the woman from 35 years, the socio-Professional group of the head of the household have a negative and significant impact on the participation of the women in agricultural activities. Thus the rich rural women of the land do not specialize in agricultural work

Résumé. Cette étude a pour but d'explorer les principaux déterminants influençant la participation des femmes dans les activités agricoles. Les données de l'enquête recueillies auprès de 678 femmes rurales vivant à l'ouest algérien. Un modèle probit a été utilisé pour estimer la contribution de chaque déterminant sur la participation des femmes rurales aux activités agricoles. Les résultats indiquent que l'âge de la femme à partir de 35 ans, la catégorie socioprofessionnelle du chef de ménage (sauf la catégorie agriculteur).
\end{abstract}

Key words: (French) femmes rurales; activités agricoles ; participation féminine; Regression logistique probit; modélisation statistique; Ouest algérien. (English) rural women; agricultural activity; participation of women; western Algeria

AMS 2010 Mathematics Subject Classification : 62Jxx; 62J05; 62J12.

JEL Subject Classification : A14, C51, R10.

\section{Presented by Professor Mamadou-Youri Sall, Université Gaston} Berger de Saint-Louis, Sénégal. Member of Editors Board.

\footnotetext{
${ }^{*}$ Corresponding author Benali Amina : amina22benaliste@yahoo.fr
} 
Benali A. African Journal of Applied Statictics, Vol. 3 (1), 2016, pages 91 - 100. Les facteurs influençant la participation des femmes rurales aux activités agricoles.

\section{Introduction}

L'objectif de cette étude est de trouver quelque réponse à des questions importantes pour les politiques socio-économiques en Algérie. Elle vise à combler un grand vide en matière d'analyse des activités agricoles. Nous tenterons notamment de répondre à une question très pertinente : Quels sont les déterminants de l'activité féminine agricole à l'ouest algérien?

Nous ferons d'abord une analyse globale de l'activité féminine en milieu rural algérien d'après les données d'une enquête de terrain. Nous donnerons quelques indicateurs de l'activité agricole des femmes rurales et de leur contribution à la vie économique. L'étude menée était à l'ouest algérien (les communes des wilayats de BECHAR, TLEMCEN TIARET, SAIDA, SIDI BEL ABBES, MASCARA, ELBAYADH, NAAMA, AIN TEMOUCHENT. Et à partir d'un échantillon composé de 678 femmes rurales, on fait une analyse des données descriptives et une analyse qualitative. Dans cette dernière, on développe un modèle probit par genre féminin, pour voir l'effet de quelque caractéristique individuelle.

\section{Cadre conceptuel et revue de littérature}

Pour comprendre le dynamisme qui caractérise les participations féminines aux activités agricoles, il s'avère indispensable de présenter et cerner quelque concept à partir des références de littératures.

De très nombreuses études socio-économiques se sont intéressées aux activités féminines et sa contribution à la vie économique ; Noté bien que le terme de femme (ou femmes) est également très controversé surtout par les chercheurs qui s'inspirent des principes de l'épistémologie constructiviste postmodern (Parini (2010)). Les critiques qui lui sont adressées plongent leurs racines dans les réflexions autour du sujet femme; sujet ontologique, politique et économique dés des années 90 (Wittig (2001), Butler (1990)).

D'une part, l'analyse de l'activité féminine a retenu l'attention des économistes avec la thèse de Friedman et Becker (1957) sur la discrimination. Du point de vue théorique, les études de Becker (1965) sur l'allocation du temps et celle de l'économie de la famille et du comportement (see Becker (1988)) mettent en évidence le travail des femmes). Depuis les années 70, on commençait à étudier les femmes au foyer et surtout les femmes rurales (Chennouf et Hafsi (2009)). En effet depuis les études de Gronau (1986), le thème des femmes au foyer actives a attiré l'attention des pouvoirs publics, avec la prise en compte dans les statistiques officielles du travail des femmes au foyer. Sa relation avec la pauvreté par exemple a été étudiée par Jackson et al. (2010).

Et d'autre part, il existe de rares travaux socio-économiques qui mettent en évidence les différentes variables qui viennent influencer la participation féminines aux activités agricoles (A partir des années 80). Il existe néanmoins des travaux américaine de ce type, leurs résultats mettent généralement en évidence un effet significatif de l'âge de la femme rurale sur l'activité agricole, tels que les travaux de Dixon-Mueller (2013) et Damisa et Yohanna (2007).

Journal home page: www.jafristatap.net 
Dans le même temps, il y a eu un regain d'intérêt pour les modèles jusqu'à l'heure actuelle tel que l'étude indienne de Pal et Haldar (2016) qui utilise un modèle probit pour analyser la participation des femmes et le processus de prise de décision dans les activités agricoles dans Katwa Block-I du district de Burdwân. Il y a également l'étude de Wittig (2001) qui utilise un modèle double-hurdle pour la production du coton en Pakistan lié aux secrets d'héritage chez les femmes.

Finalement, peu nombre d'études ont utilisé le modèle probit pour expliquer la mobilisation féminine en milieu rural, comme ceux de Yusuf et al. (2015) et Hough et al. (2015) menée aux états unis. Pour la présente étude, on développe un modèle probit d'estimation des facteurs influençant la contribution féminine au revenu de son ménage en participant aux activités agricole.

\section{Méthodologie de l'étude}

L'enquête porte sur un échantillon de 678 femmes rurales et la collecte de données se fait par un entretien personnel avec la personne concernée et le traitement se fait par le SPSS version 21 .

Plusieurs critères ont déterminé le choix des terrains sur lesquels l'enquête devait se dérouler Il importait que chaque zone rurale choisie ait une population de quelque importance Selon les statistiques de L'Office National des Statistiques d'Algérie (2011). Et pour la mise en œuvre de ce modèle, on prend en compte les critères qui ont une influence notable sur la participation des femmes rurales aux activités agricoles et extra agricoles.

(a) L'âge de la femme rurale;

(b) Le nombre de personne vivent dans le même foyer;

(c) La catégorie socioprofessionnelle du chef de ménage ;

(d) La propriété foncière.

Le modèle proposé permet de calculer de combien la prise en compte d'un effet spécifique modifie le taux d'activité moyen par rapport à la situation de référence. Les principaux facteurs influençant le taux de participation des femmes rurales aux activités agricoles sont: (Comme montre le schéma Fig. 1)

$\diamond$ L'âge de la femme rurale : l'âge de la femme rurale a un effet très marqué. Le taux de participation féminine aux activités agricoles et non agricoles (aux zones rurales) est très faible avant 25 ans, il augmente de plus en plus jusqu'à 45 ans, puis il baisse progressivement. (Voir Fig. 2).

$\diamond$ Le nombre de personnes vives dans le foyer.

On a classé les ménages en 3 catégories:

- Les ménages de petite taille: constitués de moins de 5 membres,

- Les ménages de taille moyenne: formés de 5 à 9 membres,

Journal home page: www.jafristatap.net 


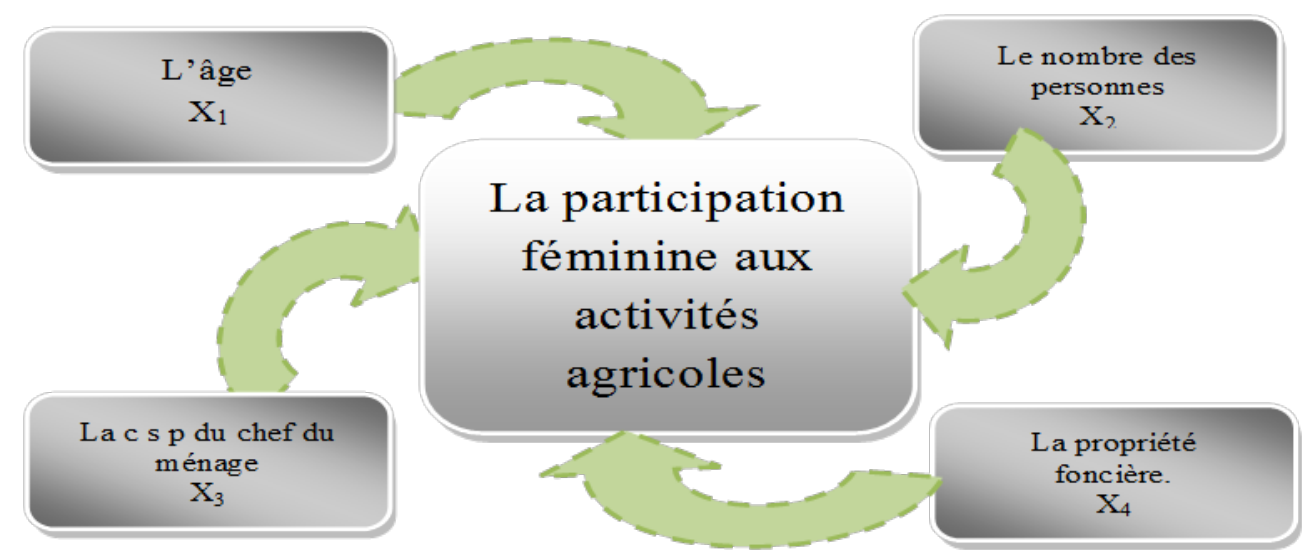

Fig. 1. Les principaux déterminants de l'étude

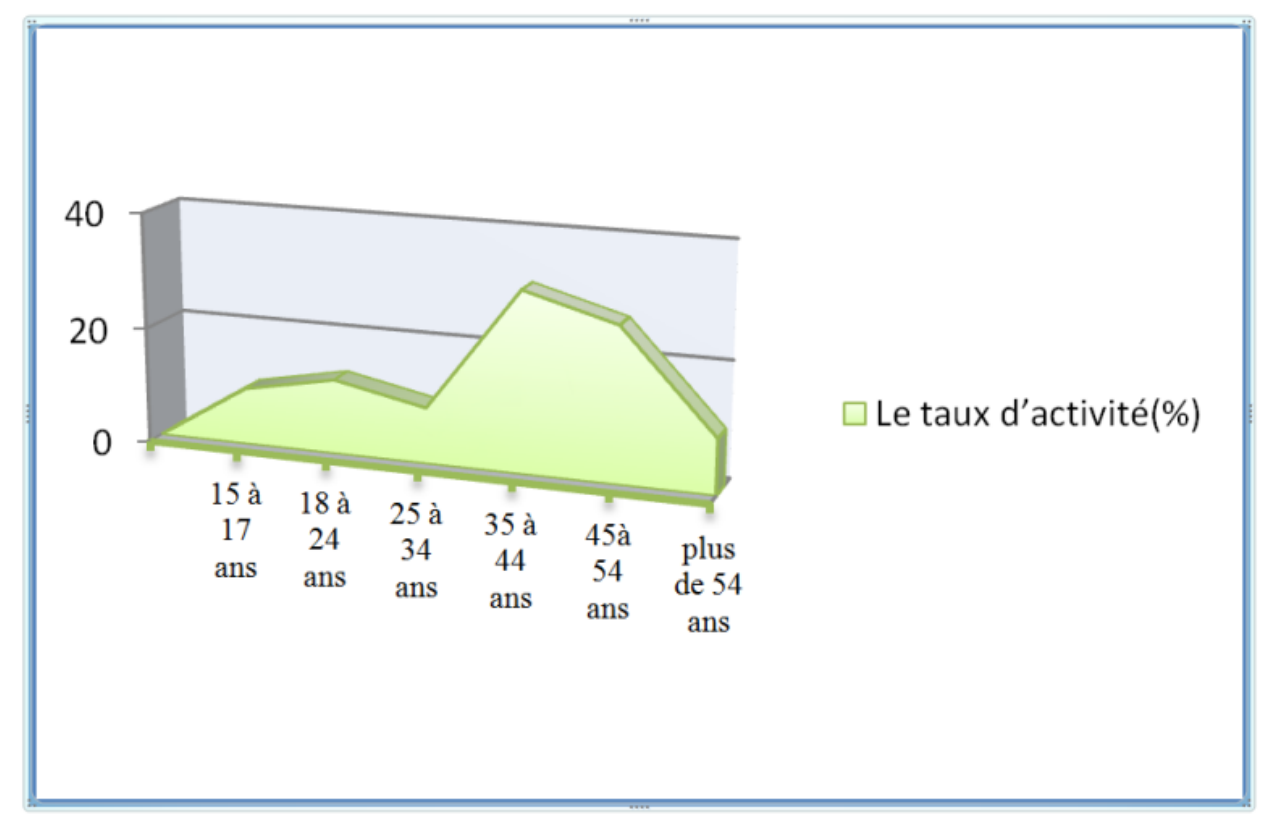

Fig. 2. Le taux de participation des femmes rurales aux activités agricoles selon leurs âges (Benali (2016))

- Les ménages nombreux: comptent plus de 10 membres.

Ce classement permet de faire ressortir qu'au niveau de l'ensemble de l'échantillon, 50, 4\% des ménages sont de grande taille (Elle dépasse la moyenne de la zone estimée à plus de 15 membres par ménage). La taille moyenne est de $22,4 \%$ de la population enquêtée. Et la petite taille est de $27,1 \%$ de la population. 
Benali A. African Journal of Applied Statictics, Vol. 3 (1), 2016, pages 91 - 100. Les facteurs influençant la participation des femmes rurales aux activités agricoles.

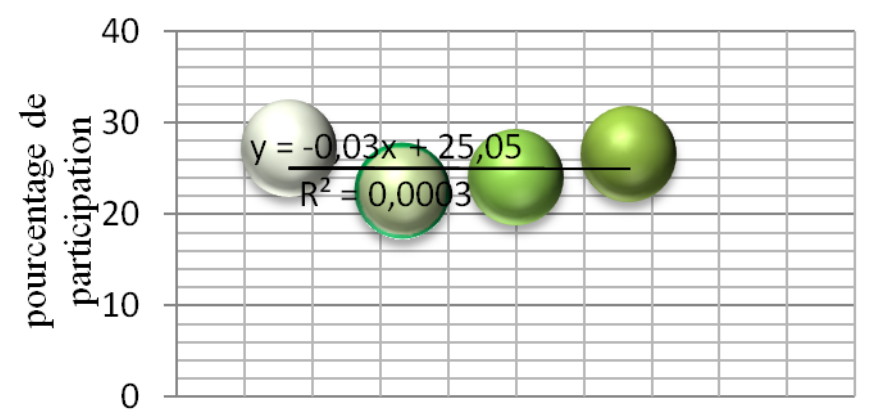

1 à4 personnes

O 5 à9 personnes

10 à 15 personnes

plus de 15 personnes

Fig. 3. Le taux de participation des femmes rurales aux activités agricoles selon leurs catégories du ménage

$\diamond$ La catégorie socioprofessionnelle du chef de ménage : les disparités sont minimes entre les femmes dont :

- Le père chef de ménage est soit sans profession;

- Le mari chef de ménage est artisan;

- Le mari chef de ménage est retraité.

Le taux de participation est plus élevé chez les femmes dont le chef de ménage est agriculteur ou un exploitant agricole (la plupart d'entre elles ont un statut d'aide familiale).

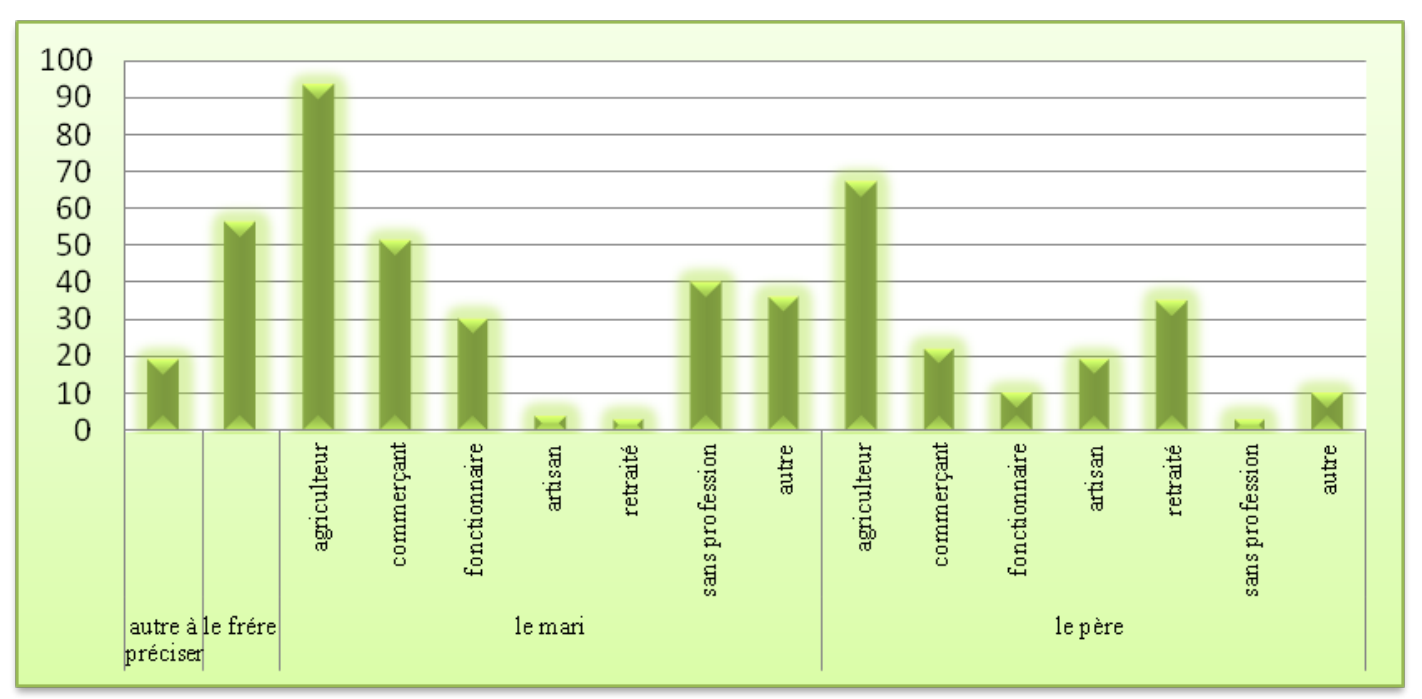

Fig. 4. Le taux de participation des femmes rurales aux activités agricole selon la catégorie socioprofessionnel du chef de ménage (Benali (2016)).

Journal home page: www.jafristatap.net 
$\diamond$ La propriété foncière:

A la question de savoir si les femmes de la zone étudiée possèdent - elles une exploitation ou une terre, la réponse a été positive pour la majorité (636femmes) partiellement héritées (375femmes affirment ça selon le tableau suivant). Et le reste a exprimé le désir d'avoir une exploitation ou une parcelle d'une terre. Elles considèrent que les terres constitue une garantie pour leur futur (en cas de divorce) mais surtout pour leurs enfants ; qu'elle est aussi un moyen d'augmenter la productivité et donc les revenus du ménage.

Quoique, la femme rurale qui a une parcelle de terre (soit par héritage, achat ou par un soutien d'état), sa probabilité d'activité est faible. La présence d'une exploitation agricole ne favorise pas la participation féminine aux activités agricoles. On remarque que l'effet de la propriété foncière est impressionnable.

\begin{tabular}{|c|r|r|r|r|r|}
\hline \multicolumn{2}{|c|}{ par } & Location & héritage & achat & $\begin{array}{c}\text { soutien } \\
\text { d'état }\end{array}$ \\
\hline $\begin{array}{c}\text { avoir une exploitation } \\
\text { agricole }\end{array}$ & OUI & 83 & 375 & 62 & 116 \\
\hline
\end{tabular}

Fig. 5. L'origine de l'exploitation agricole (Butler (1990))

Les bénéfices annuels de l'exploitation déclarée par les femmes enquêtées sont un faible niveau, selon le tableau Fig. 6, par rapport au capital investi (exploitation agricole);

\begin{tabular}{|c|c|c|c|c|c|}
\hline & & Effectifs & Pourcentage & $\begin{array}{l}\text { Pourcentage } \\
\text { valide }\end{array}$ & $\begin{array}{c}\text { Pourcentage } \\
\text { cumulé }\end{array}$ \\
\hline \multirow[t]{6}{*}{ Valide } & entre 100000 et $500000 \mathrm{DA}$ & 4 & 6 & 1,1 & 1,1 \\
\hline & $\begin{array}{c}\text { entre } 500000 \text { et } 1000000 \\
\text { DA }\end{array}$ & 112 & 16,5 & 29,5 & 30,5 \\
\hline & $\begin{array}{c}\text { entre } 1000000 \text { et } 5000000 \\
\text { DA }\end{array}$ & 46 & 6,8 & 12,1 & 42,6 \\
\hline & $\begin{array}{c}\text { entre } 5000000 \text { et } 10000000 \\
\text { DA }\end{array}$ & 122 & 18,0 & 32,1 & 74,7 \\
\hline & Plus de $10000000 \mathrm{DA}$ & 96 & 14,2 & 25,3 & 100,0 \\
\hline & Total & 380 & 56,0 & 100,0 & \\
\hline \multicolumn{2}{|c|}{ N'ont pas une exploitation } & 298 & 44,0 & & \\
\hline \multicolumn{2}{|l|}{ Total } & 678 & 100,0 & & \\
\hline
\end{tabular}

Fig. 6. Les bénéfices annuels de 'exploitation agricole (Benali (2016)) 


\section{Présentation du modèle d'analyse}

Dans cette étude, on utilise le modèle probit dont on suppose que la participation féminine aux activités agricoles est une fonction de : l'âge de la femme rurale (X1), le nombre de personne vivent dans le même foyer $(X 2)$, la catégorie socioprofessionnelle du chef de ménage $(X 3)$ et la propriété foncière de la femme rurale $(X 4)$.

Donc on peut écrire : l'ensemble des variables représentant les facteurs qui semblent a priori avoir une influence importante sur la participation des femmes rurales aux activités agricoles est :

$$
\begin{aligned}
& \mathrm{X}_{1}\left[\begin{array}{l}
x_{11}: \text { de } 15 \grave{a} 17 \text { ans } \\
x_{12}: \text { de } 18 \text { à } 24 \text { ans } \\
x_{13}: \text { de } 25 \grave{a} 34 \text { ans } \\
x_{14}: \text { de } 35 \text { à } 44 \text { ans } \\
x_{15}: \text { de } 45 \grave{a} 54 \text { ans } \\
x_{16}: \text { plus de } 54 \text { ans }
\end{array}\right]
\end{aligned}
$$

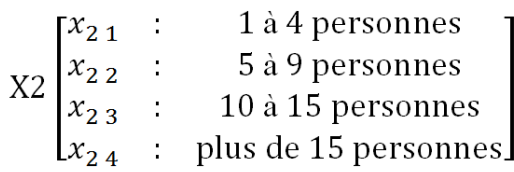

$$
\begin{aligned}
& X_{3}\left[\begin{array}{ccc}
x_{31} & : & \text { agriculteur ou exploitant } \\
x_{32}: & \text { commercant } \\
x_{33} & : & \text { fonctionnaire } \\
x_{34}: & \text { artisan } \\
x_{35}: & \text { retraité } \\
x_{36}: & \text { sans prefession } \\
x_{37}: & \text { autre }
\end{array}\right] \quad X 4\left[\begin{array}{ccc}
x_{41}: & \text { heritage } \\
x_{42}: & \text { achat } \\
x_{43}: & \text { location } \\
x_{44}: & \text { soutien d'état }
\end{array}\right]
\end{aligned}
$$

On dispose pour chaque femme rurale (i) de l'échantillon étudiée des valeurs $\left(x_{1,1}, \cdots, x_{1,678}\right)$ prise par l'âge, les valeurs $\left(x_{2,1}, \cdots, x_{2,678}\right)$, prise par le nombre de personnes vivant dans le même foyer; etc.

On suppose que chaque femme rurale ait la probabilité de participer aux activités agricoles avec un taux précis, donc on a :

$$
p\left(y_{i}=1\right)=F\left({ }^{t} \ell x_{i},\right)
$$

où :

$i$ varie entre 1 et 678 ;

$P$ est la probabilité de participation;

$y$ est une variable, qui pour chaque femme rurale prend la valeur 1 quand elle participe aux activités agricoles et vice-versa.

Journal home page: www.jafristatap.net 
$F$ : est la fonction de répartition de la loi normale et ou ${ }^{t} \ell x_{i}$ désigne la forme linéaire

$$
{ }^{t} \ell x_{i}=\ell_{(}(0)+\sum_{j} \ell_{j}
$$

Si l'échantillon utilisé comporte $\mathrm{n}$ femmes rurales $(n=678)$, et que les observations sont indépendantes, la probabilité d'obtenir les n participations s'écrit :

$$
p=\prod_{678}^{i=1}\left(F\left({ }^{t} \ell x_{i}\right)\right)^{y_{i}}\left(\left(1-F\left({ }^{t} \ell x_{i}\right)\right)\right)^{1-y_{i}}
$$

L'estimation des $(p+1)$ coefficients $\ell_{j}$ se fait par la méthode du maximum de vraisemblance : on retient les valeurs des coefficients $\ell_{j}$ qui maximisent le membre de droite de l'équation ci-dessus.

Et on suppose que toutes les variables explicatives ont été mises sous forme dichotomique. Tout d'abord, les variables quantitatives, âge de la femme, le nombre de personne, ont été transformées en variables qualitatives par découpage en tranches. Ensuite, pour chaque caractéristique, une variable dichotomique a été associée à chaque modalité.

Ainsi, pour la catégorie socioprofessionnelle du chef de ménage, sept modalités ont été distinguées, auxquelles correspondent sept variables : la première vaut 1 si le chef de ménage est agriculteur, 0 dans le cas contraire; la seconde vaut 1 si le chef de ménage est commerçant, 0 dans le cas contraire; et ainsi de suite.

L'ensemble des modalités non introduites, une par caractéristique, constitue une situation de référence, pour laquelle la probabilité d'être active est égale à $F\left(\ell_{0}\right)$. Si l'on change une modalité d'une caractéristique, modalité à laquelle correspondent la variable $X_{J}$, la probabilité devient $F\left(l 0+\ell_{j}\right)$.

Les valeurs estimées des coefficients lj sont données dans le tableau de Fig. 7.

Globalement, la participation féminine aux activités agricoles a un effet positif chez la femme rurale de 15 à 34 ans, qui cohabite dans un ménage de moyenne taille, et son chef de ménage est agriculteur ; par contre complètement chez la femme rurale de 35 ans ou plus, qu'elle cohabite dans un ménage multi nucléaire (la troisième catégorie), et son chef de ménage est un retraité.

\section{Conclusion}

Nous devons préciser que notre analyse est basée sur les données d'une enquête réalisée dans quelque wilaya d'Algérie, et en raison de la grande dimension du pays, il existe des disparités importantes entre les diverses wilayas. La participation féminine aux activités agricoles pourrait varier d'une wilaya à l'autre et d'une zone rurale à autre .Par ailleurs, il serait intéressant d'étudier la différentiation de l'effet de l'activité agricole selon les divers sous-groupes des ménages. Par ailleurs, notre analyse économétrique met en lumière l'effet 


\begin{tabular}{|l|l|}
\hline \multicolumn{2}{|c|}{ Participation aut activités agricoles } \\
\hline L'âge de l'interviewée: \\
\hline De 15 à 17 ans & 1,038 \\
\hline De 18 à 24 ans & 0,458 \\
\hline De 25 à 34 ans & $1,120^{*}$ \\
\hline De 35 ans à 44 ans & 0,587 \\
\hline De 45 ans à 54 ans & $-0,519$ \\
\hline Plus de 54 ans & $-0,683$ \\
\hline Le nombre de personne /foyer & \\
\hline De 1 à 4 personnes & 0,982 \\
\hline De 5 à 9 personnes & $1,541^{*}$ \\
\hline De 10 à 15 personnes & 0,532 \\
\hline Plus de 15 personnes & $-0,054$ \\
\hline Cs p du chef de ménage: & \\
\hline Agriculteur ou exploitant agricole & $1,768^{*}$ \\
\hline Commerçant & 0,317 \\
\hline Fonctionnaire & 0,447 \\
\hline Artisan & 0,272 \\
\hline Retraité & $-0,151$ \\
\hline Sans profession & 0,931 \\
\hline La propriété foncière: & 0,741 \\
\hline héritage & 0,054 \\
\hline Achat & $-0,791$ \\
\hline Location & \\
\hline Soutien d'état & 1,885 \\
\hline & \\
\hline
\end{tabular}

Fig. 7. Estimations de paramètres

des divers facteurs sur l'activité agricole dans la région retenue.

Premièrement, nos résultats révèlent que la participation aux diverses activités est toujours positivement liée au nombre des personne vivent dans le même foyer. Deuxièmement, notre analyse confirme le rôle important que joue l'agriculteur chef de ménage dans la prise de décision de la participation féminine à l'activité purement agricole. Enfin, nos résultats montrent que les femmes relativement riches en terre ne se spécialisent pas forcément dans la production agricole.

\section{References}

Becker, G. S, (1965). A Theory of the Allocation of Time. The economic journal. 493-517. 
Becker, G. S. (1988). Family Economics and Macro Behavior. The American Economic Review.

Benali A.(2016). Le concept de genre et la contribution de la femme rurale au revenue de son ménage dans les zones rurales et les exploitations agricoles, these de doctorat, université de Tlemcen.

Butler, J.(1990). Gender Trouble: Feminist Theory, and Psychoanalytic Discourse. Feminism/Postmodernism. New York/Londres, Routledge, 324-340.

Chennouf, S. et Hafsi, T. (2009). Entrepreneurs et salaries en Algérie: Application d'un probit bivarié par genre et par secteur. Les cahiers du Cread, 89, 23-51.

Damisa, M. A. et Yohanna, M. (2007). Role of rural women in farm management decision making process: ordered probit analysis. World Journal of Agricultural Sciences, 3(4), 543-546.

Dixon-Mueller, R. B. (2013). Rural women at work: Strategies for development in South Asia. Routledge.

Friedman, M., et Becker, G. S.(1957). A statistical illusion in judging Keynesian models. The journal of political economy, 64-75.

Gronau, R.(1986). Home production-a survey. Handbook of labor economics, 1, 273-304.

Hough, J., Cao, J., et Handy, S. (2015). Relative Desired Mobility of Elderly Women Living in Rural and Small Urban Locations in North Dakota. In: Transportation Research Board, 94th Annual Meeting, no. 15-4559.

Jackson, L. R., Dip, B. A., et Jackson, L.(2010). Influences on financial decision making among the rural poor in Bangladesh. University of Western Sydney.

Kouser, S., et Qaim, M.(2015). Cotton and employment effects for female agricultural laborers in Pakistan: An application of double-hurdle model. In the International Conference of Agricultural Economists (ICAE) (pp. 8-14).

Parini L.(2010). Le concept de genre: constitution d'un champ d'analyse, controverses épistémologiques, linguistiques et politiques.Socio-logos. Revue de l'association française de sociologie [En ligne],5, mis en ligne le 07 juillet 2010. URL : http://sociologos.revues.org/2468, p5-6

Office National de la Statistique .(2011) Les statistiques des enquêtes d'emploi et des ménages. Alger.

Pal, S. et Haldar, S.(2016). Participation and role of rural women in decision making related to farm activities: A study in Burdwan district of West Bengal. Economic Affairs, 61(1), 55.

Soheil ....

Wittig, M.(2001). La pensée straight. Paris, Editions Balland.

Yusuf, H. A., Nuhu, K. J., Shuaibu, H., Yusuf, H. O. and Yusuf, O. (2015). Factors Affecting the Involvement of Women in Income Generating Activities in Sabon-Gari Local Government Area of Kaduna State, Nigeria. American Journal of Experimental Agriculture, 5(1), 54. 